\title{
FEDERALISMO E MERIDIONALISMO NEL CRISTO SI È FERMATO A EBOLI DI CARLO LEVI
}

\section{Alberto Vespaziani ${ }^{1}$}

\begin{abstract}
RIASSUNTO: Questo contributo si concentra sul rapporto tra cultura federale e problema meridionale in un testo classico della letteratura italiana, il Cristo si è fermato a Eboli di Carlo Levi. Il discorso si articola in quattro parti: dapprima riassume il carattere centralista della cultura giuridica dominante in Italia, quindi ricorda la genesi e il contenuto del capolavoro di Levi; nella terza parte evidenzia i temi politico-costituzionali che emergono dal romanzo, infine conclude discutendo il rapporto ambivalente tra i vari tipi di censura che colpirono l'autore e la genealogia eroica del suo racconto.
\end{abstract}

PARole-Chiave: Italia; federalismo; meridionalismo; minoranze; libertà; creatività; censura.

\section{CULTURA FEDERALE E PROBLEMA MERIDIONALE IN ITALIA}

La cultura giuridico-politica dominante dell'Italia unita è stata caratterizzata da un'impronta centralistica. Prima dell'unificazione vi erano state correnti politiche e pensatori che avevano vagheggiato un processo di unificazione di tipo federale, tuttavia rimasero minoritari. L'unità politica italiana è nata nel peccato originale centralistico, e da allora, per una sorta di path-dependency, le dinamiche politiche si sono sempre articolate all'interno del discorso dominante del centralismo statalista (Vespaziani, 2013). Alla iniziale piemontesizzazione è succeduto il centralismo fascista; l'incerto sussulto regionalista del processo costituente post-bellico è stato presto svuotato dalla burocratizzazione, corruzione e clientelismo che hanno trascinato le regioni italiane (con qualche rara eccezione) nello stato

1 Dottore in Diritto Costituzionale presso l'Università La Sapienza di Roma. LL. M. (Harvard). Professore Associato di Diritto Pubblico Comparato nell'Università degli Studi del Molise (UNIMOL). Molise, Italia. E-mail: alberto.vespaziani@unimol.it 
comatoso in cui oggi si trovano. Se a ciò aggiungiamo il centralismo partitocratico della c.d. Prima Repubblica (1948-1992) ed il centralismo mediatico della c.d. Seconda Repubblica (1994-?) vediamo come il centralismo abbia saputo assorbire i vari assetti del potere che la lotta politica italiana ha articolato nel secondo dopoguerra.

$\mathrm{Su}$ queste macerie della cultura politica federale italiana è forse possibile tornare a volgere lo sguardo a quella ricca tradizione giuridica meridionalista, che in vari fasi della storia d'Italia aveva invocato la soluzione federalista per risolvere il problema della spaccatura nord-sud, che a partire dall'unificazione non si è mai attenuata. Non vi è dubbio che parlare di federalismo meridionalista significa invocare una corrente minoritaria ed anti-egemonica all'interno del panorama politico-giuridico italiano; l'argomento ricorrente, il leit-motiv della cultura centralista italiana, ripetuto al tempo dell'unificazione, del fascismo, dell'assemblea costituente e della riforma costituzionale del 2001 è sempre stato "il Sud non è pronto". L'idea secondo cui una trasformazione in senso federale delle istituzioni politiche non può che aggravare il divario socio-economico tra settentrione e meridione è stato periodicamente riproposto. In attesa di una verifica contro-fattuale, l'unica certezza pare essere la inidoneità/impotenza degli assetti centralistici ad affrontare la questione principale della spaccatura tra parti avvantaggiate del paese e parti arretrate, che non accenna a diminuire.

Invocando il pensiero delle possibilità (Häberle, 1996, p. 19), questo articolo richiama l'attenzione sul tesoro nascosto della cultura giuridica meridionalista italiana, a partire da un'opera letteraria: il Cristo si è fermato a Eboli, di Carlo Levi. Levi non era un pensatore politico in senso stretto, tuttavia le sue esperienze di lotta e resistenza al fascismo lo avevano portato ad avvicinarsi ad autori federalisti italiani, mentre la persecuzione del regime lo condusse ad esplorare e ad immergersi nei territori più arretrati dell'Italia meridionale.

\section{GENESI E CONTENUTO DEL CRISTO SI ל̀ FERMATO A EBOLI DI CARLO LEVI: LE CENSURE DELLA LOTTA POLITICA E DELLA STORIA DEL MONDO}

Carlo Levi era nato a Torino nel 1902, dove si era laureato in medicina nel 1923, ma pur essendo stato educato ad una disciplina scientifica in un 
ambiente culturale intriso di positivismo, ben presto trascurò la professione medica e la carriera universitaria per dedicarsi con successo alla pittura. Cresciuto negli ambienti raffinati della borghesia ebraica torinese, egli trascorse un'infanzia serena ed una giovinezza agiata, con frequenti visite a Parigi (Bartalesi-Graf, 2006, p. 514).

Con l'avvento del fascismo, Levi entrò in sintonia con Pietro Gobetti ed il suo socialismo liberale, ed insieme a Salvemini, Lussu, Rosselli e Tarchiani fondò il movimento "Giustizia e libertà". Ben presto la sua attività pubblicistica e politica destò le attenzioni della polizia fascista che lo arrestò una prima volta nella elegante villa di famiglia di Alassio nel 1934 e lo tradusse nelle carceri di Torino dove rimase per quasi due mesi.

Per nulla intimidito da quella esperienza, Levi riprese la sua attività politica di contrasto al regime, che lo fece di nuovo arrestare il 15 maggio del 1935, trasferire nel carcere di Regina Coeli a Roma, dove due mesi dopo venne condannato dalla Prefettura di Roma al confino di polizia per tre anni, destinazione Grassano, un paesino sconosciuto della Lucania, "per avere dato adesione ed attività ad un'associazione segreta denominata 'Giustizia e Libertà' avente lo scopo di commettere fatti diretti a mutare violentemente la costituzione dello Stato e la forma di Governo" (De Donato; D’amaro, 2005, p. 116).

Fu così che in piena estate Carlo Levi si inoltrò per la prima volta in vita sua nelle terre del profondo Mezzogiorno d'Italia. Dopo pochi mesi egli venne trasferito ad Aliano, un paesino ancora più sperduto (King; Killingbeck, 1989, p. 132), dove trascorse dieci mesi, in cui tradusse lo sconvolgente incontro con un'umanità arcaica in immagini pittoriche e poesie, ma non in racconti o appunti letterari, visto che era strettamente sorvegliato, e tutta la sua corrispondenza veniva controllata dal podestà locale, nonché dal prefetto di Matera. In occasione della conquista di Adis Abeba e della proclamazione dell'Impero, il governo italiano dispose la liberazione di molti confinati politici, tra cui Carlo Levi, che lasciò Aliano il 26 maggio del 1936.

Tornato a Torino, fu costretto all'esilio dalla entrata in vigore delle leggi razziali e nel giugno del 1939 si rifugiò prima a Parigi, poi a Cannes. Pur avendo acquistato un biglietto navale da Lisbona a New York, Levi rinunciò a partire per gli Stati Uniti e nell'estate del 1941, "con il cuore tutto 
dalla parte dell'Italia" (De Donato; D’amaro, 2005, p. 146) decise di rientrare in patria, a Firenze, dove nella primavera del '43 venne nuovamente arrestato e incarcerato, per essere poi liberato il 25 luglio del 1943, giorno della caduta del regime fascista.

Nella Firenze invasa dai tedeschi Levi entrò in clandestinità e si rifugiò presso uno dei suoi amori, Annamaria Ichino, in Piazza Pitti. "Chiuso in una stanza, e in un mondo chiuso" (Levi, 2014, p. 3), mentre la tragedia della guerra civile e dell'occupazione nazista incombevano sulla città, Levi iniziò a scrivere il suo primo romanzo, il Cristo si è fermato a Eboli, in cui ricostruì le memorie di quei dieci mesi di confino dove aveva scoperto la "Lucania che è in ciascuno di noi" (Levi, 2014, p. xviii), la povertà e la miseria del Sud Italia, ma anche quel luogo periferico ed emarginato che consente ad ogni individuo di sottrarsi alle tendenze narcisistiche per sperimentare la vera empatia e solidarietà umana.

Questa sintesi della genealogia dell'opera serve ad evidenziare il paradossale effetto liberatorio e creativo che le costrizioni censorie hanno operato sulla mentalità e sull'opera di un pittore/dottore: solo il ripetersi dell'esperienza della reclusione forzata rese possibile la traduzione dell'esperienza del confino in un racconto, "come per un processo di cristallizzazione amorosa" (Levi, 2014, p. xix), (come Levi scrisse nel 1963 in una lettera al suo editore Giulio Einaudi).

Il titolo del libro, suggeritogli successivamente dal contadino anarchico Michele Mulieri (Coccia, 2015, p. 289), riprende un modo di dire dei contadini lucani, che simboleggia il profondo isolamento delle terre interne dell'appennino meridionale, dove non erano giunti né i greci né $\mathrm{i}$ romani, dove le teocrazie statali dei piemontesi e dei fascisti erano rimaste estranee alla vita dei contadini, dove neanche il cristianesimo era riuscito a redimere le popolazioni meridionali dalla loro secolare disperazione. Ad Eboli, una città poco a sud di Salerno, si era dunque fermata la Storia umana, e le persone vivevano il tempo eterno della violenza della natura e della immutabilità delle relazioni sociali.

Il romanzo racconta dunque gli incontri che Carlo Levi fece con la piccola borghesia locale, che egli disprezzava per la sua mediocrità, rapacità e rassegnazione, e con i contadini, con il cui mondo magico e disperato egli entrò in profonda sintonia. 
La borghesia rurale degenerata era composta da magistrati, avvocati, medici, impiegati, che vivevano all'ombra della grande proprietà fondiaria, che subivano e allo stesso tempo riverivano. Questi soggetti avevano un atteggiamento di disprezzo, di odio, di prepotenza nei confronti dei contadini, cui negavano diritti e persino l'uso della parola, riducendoli al silenzio e all'impotenza, chiusi nel loro mondo oscuro senza speranza. Così Levi descrive le sue prime impressioni all'arrivo nello sperduto borgo:

mi avevano colpito il sussiego, le maniere dei signori sulla piazza, e più ancora il tono generale di astio, disprezzo e diffidenza reciproca nella conversazione a cui avevo assistito, la facilità con cui si manifestavano degli odi elementari, senza il naturale ritegno verso un forestiero appena arrivato, che aveva fatto sì che io fossi messo subito al corrente da ciascuno dei vizi o delle debolezze degli altri. Per quanto non potessi ancora determinarlo con esattezza, era chiaro che anche qui, come a Grassano, gli odi reciproci di tutti contro tutti si cristallizzavano in due partiti. Qui, come a Grassano, come in tutti gli altri paesi della Lucania, dove i galantuomini che non hanno potuto, per incapacità o povertà, o matrimoni precoci, o interessi da tutelare, o per una qualunque necessità del destino, emigrare ai paradisi di Napoli o di Roma, trasformano la propria delusione e la propria noia mortale in un furore generico, in un odio senza soste, in un perenne risorgere di sentimenti antichi, e in una lotta continua per affermare, contro tutti, il loro potere nel piccolo angolo di terra dove sono costretti a vivere [...]. La piccola borghesia non ha mezzi sufficienti per vivere col decoro necessario, per fare la vita del galantuomo. Tutti i giovani di qualche valore, e quelli appena capaci di fare la propria strada, lasciano il paese. I più avventurati vanno in America, come i cafoni; gli altri a Napoli o a Roma; e in paese non tornano più. In paese ci restano invece gli scarti, coloro che non sanno far nulla, i difettosi nel corpo, gli inetti, gli oziosi: la noia e l'avidità li rendono malvagi. Questa classe degenerata deve, per vivere (i piccoli poderi non rendono quasi nulla), poter dominare i contadini, e assicurarsi, in paese, i posti remunerati di maestro, di farmacista, di prete, di maresciallo dei carabinieri, e così via. È dunque questione di vita o di morte avere personalmente in mano il potere; essere noi o i nostri parenti o compari ai posti di comando. Di qui la lotta continua per arraffare il potere tanto necessario e desiderato, e toglierlo agli altri; lotta che la ristrettezza dell'ambiente, l'ozio, l'associarsi di motivi privati o politici rende continua e feroce (Levi, 2014, p. 20). 
Ma oltre la meschinità dell'ideologia piccolo-borghese, Levi scoprì ad Aliano la sostanza politica della questione meridionale (Ragusa, 1969, p. 337). È quindi ai contenuti politico-costituzionali dell'opera che si rivolge ora l'attenzione.

\section{LA QUESTIONE MERIDIONALE COME PROBLEMA COSTITUZIONALE: IL FEDERALISMO CENSURATO}

Come mai nella tradizione giuridica dell'Italia unita il federalismo è sempre rimasto una cultura minoritaria e sconfitta? Quali sono le ragioni del persistere della rappresentazione dello stato come di una forza esterna, arbitraria e violenta, presso la mentalità diffusa delle popolazioni meridionali italiane? E perché la borghesia meridionale ha sempre preferito il contatto con il potere centrale di Roma rispetto ai rapporti con le comunità locali? Questi interrogativi si agitano nel Cristo e generano sia narrazioni commoventi sia risposte politico-costituzionali.

Per Levi la borghesia locale, incapace e corrotta, aveva la necessità di essere vicina al potere, per potersi assicurare gli uffici pubblici e per poter dominare i contadini, ed essendo i posti pubblici disponibili scarsi, la lotta per ottenerli era feroce, contribuendo ad inacidire gli animi rancorosi. Perfino durante il periodo del regime fascista, nessun contadino si era iscritto al partito; per i contadini la politica rimaneva estranea e lo stato nemico. Così Levi descrive il diverso livello di adesione al regime fascista:

I signori erano tutti iscritti al Partito, anche quei pochi, come il dottor Milillo, che la pensavano diversamente, soltanto perché il Partito era il Governo, era lo Stato, era il Potere, ed essi si sentivano naturalmente partecipi di questo potere. Nessuno dei contadini, per la ragione opposta, era iscritto, come del resto non sarebbero stati iscritti a nessun altro partito politico che potesse, per avventura, esistere. Non erano fascisti, come non sarebbero stati liberali o socialisti o che so io, perché queste faccende non li riguardavano, appartenevano a un altro mondo, e non avevano senso. Che cosa avevano essi a che fare con il Governo, con il Potere, con lo Stato? Lo Stato, qualunque sia, sono «quelli di Roma», e quelli di Roma, si sa, non vogliono che noi si viva da cristiani. C'è la grandine, le frane, la siccità, la malaria, e c'è lo Stato. Sono dei mali inevitabili, ci sono sempre stati e ci saranno sempre. Ci fanno ammazzare le capre, ci portano via i mobili di casa, e adesso ci manderanno a fare la guerra. 
Pazienza! Per i contadini, lo Stato è più lontano del cielo, e più maligno, perché sta sempre dall'altra parte. Non importa quali siano le sue formule politiche, la sua struttura, i suoi programmi. I contadini non li capiscono, perché è un altro linguaggio dal loro, e non c'è davvero nessuna ragione perché li vogliano capire. La sola possibile difesa, contro lo Stato e contro la propaganda, è la rassegnazione, la stessa cupa rassegnazione, senza speranza di paradiso, che curva le loro schiene sotto i mali della natura (Levi, 2014, p. 67).

Qualsiasi forma di potere pubblico si era addentrato nei secoli in quelle terre solo per sfruttare violentemente i contadini. Terra di emigrazione, la Lucania non guardava più a Napoli, oramai senza re né futuro, solo un porto dove imbarcarsi su un piroscafo per raggiungere l'America, né a Roma, "la capitale dei signori, il centro di uno Stato straniero e malefico" (Levi, 2014, p. 108). Se i contadini senza stato potessero avere una capitale loro, così Levi, questa sarebbe New York. Chi ha potuto, è emigrato là, chi è tornato, per nostalgia o perché ha ingenuamente risposto alla propaganda del regime che invitava a tornare nell'Italia imperiale con la prospettiva di ottenere terre fertili in Africa, vive nel rimorso e nel ricordo mitizzato del tempo lì trascorso.

Nel penultimo capitolo del Cristo, Levi racconta un episodio cruciale, di quando durante il periodo di confino ottiene un permesso per recarsi a Torino in occasione della morte di un suo stretto parente, ed incontra gli amici intellettuali e politici. Trasformato nel profondo dalla sua esperienza ad Aliano, egli si accorge che non riesce più a comunicare con coloro i quali condivideva le passioni politiche:

Tutti mi avevano chiesto notizie del mezzogiorno; a tutti avevo raccontato quello che avevo visto: e, se tutti mi avevano ascoltato con interesse, ben pochi mi era parso volessero realmente capire quello che dicevo. Erano uomini di varie opinioni e temperamenti: dagli estremisti più accesi ai più rigidi conservatori. Molti erano uomini di vero ingegno e tutti dicevano di aver meditato sul «problema meridionale» e avevano pronte le loro formule e i loro schemi. Ma così come queste loro formule e schemi, e perfino il linguaggio e le parole usate per esprimerli sarebbero stati incomprensibili all'orecchio dei contadini, così la vita e i bisogni dei contadini erano per essi un mondo chiuso, che neppure si curavano di penetrare. Erano, in fondo, tutti (mi pareva ora di vederlo chiaramente) degli adoratori, più o meno inconsapevoli, dello Stato; degli idolatri che si ignoravano. Non importava se il loro Stato fosse quello attuale o quello che 
vagheggiavano nel futuro: nell'uno e nell'altro caso era lo Stato, inteso come qualcosa di trascendente alle persone e alla vita del popolo; tirannico o paternamente provvidente, dittatoriale o democratico, ma sempre unitario, centralizzato e lontano. Di qui la impossibilità, fra i politici e i miei contadini, di intendere e di essere intesi (Levi, 2014, p. 219).

I politici tutti, conservatori e progressisti, credevano che il problema meridionale fosse una parte del problema nazionale, e che solo con un intervento più forte dello stato sarebbe stato possibile ricondurre le popolazioni del sud entro la vita politica unitaria. Per Levi viceversa

lo Stato, come essi lo intendevano, era invece l'ostacolo fondamentale a che si facesse qualunque cosa. Non può essere lo Stato [...] a risolvere la questione meridionale, per la ragione che quello che noi chiamiamo problema meridionale non è altro che il problema dello Stato (Levi, 2014, p. 220).

Rovesciando la rappresentazione tradizionale del rapporto nord-sud come di una relazione tra normalità istituzionale ed eccezionalità criminale, Levi mostra come la questione meridionale riveli l'insufficienza del processo di unificazione costituzionale avvenuto secondo modalità centralistiche. Il problema del sud è insomma il problema della mancanza iniziale di legittimazione politico-sociale delle strutture istituzionali e della debolezza o assenza di cultura costituzionale comune, di senso di appartenenza ad una comune unità politica.

Per uscire dall' "eterno fascismo italiano" (Levi, 2014, p. 222), Levi ritiene che "bisogna che noi ci rendiamo capaci di pensare e di creare un nuovo Stato, che non può più essere né quello fascista, né quello liberale, né quello comunista, forme tutte diverse e sostanzialmente identiche della stessa religione statale. Dobbiamo ripensare ai fondamenti stessi dell'idea di Stato: al concetto d'individuo che ne è la base; e, al tradizionale concetto giuridico e astratto di individuo, dobbiamo sostituire un nuovo concetto, che esprima la realtà vivente, che abolisca la invalicabile trascendenza di individuo e di Stato. L'individuo non è una entità chiusa, ma un rapporto, il luogo di tutti i rapporti. Questo concetto di relazione, fuori della quale l'individuo non esiste, è lo stesso che definisce lo Stato. Individuo e Stato coincidono nella loro essenza, e devono arrivare a coincidere nella pratica quotidiana, per esistere entrambi. Questo capovolgimento della politica, che 
va inconsapevolmente maturando, è implicito nella civiltà contadina, ed è l'unica strada che ci permetterà di uscire dal giro vizioso di fascismo e antifascismo. Questa strada si chiama autonomia. Lo Stato non può essere che l'insieme di infinite autonomie, una organica federazione" (Levi, 2014, p. 223).

Questa concezione costituzionale era giunta a Levi mediante il contatto con autori di origini meridionali quali il pugliese Gaetano Salvemini, il sardo Emilio Lussu ed il campano Guido Dorso. Pur divergendo sulle concezioni generali del federalismo (più amministrativo e fiscale quello di Salvemini, costituzionale quello di Lussu e politico quello di Dorso), nonché sul livello territoriale più adeguato a costituire l'entità federata di riferimento (le regioni per Lussu, le città per Salvemini), i tre autori qui rievocati erano concordi sull'importanza del magistero di Carlo Cattaneo, e quindi consideravano una sciagura la mancata unificazione secondo dinamiche federali degli Stati Uniti d'Italia. Essi erano d'accordo sul fatto che l'unificazione centralizzatrice, la conquista regia del sud e la piemontesizzazione forzata del meridione, avevano gettato le basi per un rigetto del senso di comune appartenenza, per un esproprio delle ricchezze del sud a favore del nord e per il consolidamento di politiche trasformiste e clientelari che non sarebbe più stato possibile debellare.

La visione federale che aveva condiviso con Lussu, Olivetti e Dorso diventa in Levi una proposta utopica, basata su di un'esperienza drammaticamente reale, per rovesciare l'egemonia della cultura centralistica, adoratrice dello stato, di qualsiasi forma di stato, causa prima del distacco progressivo delle masse contadine meridionali dalle istituzioni politiche. In un’intervista del 1973 così Levi si espresse:

direi che valgono, esistono soltanto le cose che si realizzano completamente, nella propria autonomia. Quelle invece che sono apparenze, non si realizzano in quel senso, anzi impediscono lo sviluppo autonomo delle forze reali [...] L'ho anche teorizzato talvolta; per esempio in tutti i miei scritti politici clandestini che stampavo sui «Quaderni di Giustizia e Libertà» dove appunto ho sviluppato i concetto di autonomia anche come progetto come utopia di un futuro Stato libero dopo le lotte contro il fascismo (Romano, 1985, p. 87). 
Il problema della forma di stato, democratica o autoritaria, centralistica o federale, non è per Levi tuttavia confinabile all'interno del paradigma statalista: le forme politiche fondamentali della vita in comune sono nel meridione italiano determinate da un vero e proprio scontro di civiltà, quello tra vita borghese e vita contadina: "siamo anzitutto di fronte al coesistere di due civiltà diversissime, nessuna delle quali è in grado di assimilare l'altra. Campagna e città, civiltà precristiana e civiltà non più cristiana, stanno di fronte; e finché la seconda continuerà ad imporre alla prima la sua teocrazia statale, il dissidio continuerà. La guerra attuale, e quelle che verranno, sono in gran parte il risultato di questo dissidio secolare, giunto ora alla sua più intensa acutezza, e non soltanto in Italia. La civiltà contadina sarà sempre vinta, ma non si lascerà mai schiacciare del tutto, si conserverà sotto i veli della pazienza, per esplodere di tratto in tratto; e la crisi mortale si perpetuerà. Il brigantaggio, guerra contadina, ne è la prova: e quello del secolo scorso non sarà l'ultimo. Finché Roma governerà Matera, Matera sarà anarchica e disperata, e Roma disperata e tirannica" (Levi, 2014, p. 221).

\section{LE CENSURE STATALISTICHE E LA NATURA CONTRO- EGEMONICA DELLA CULTURA FEDERALISTA IN ITALIA}

Vista dal nord, la questione meridionale appare come un problema relativo solo alle persone che vivono nei territori del sud Italia; osservata da Aliano, viceversa, essa si presenta nella sua sostanza politica come l'insufficienza del processo centralistico di unificazione costituzionale. La persistente ammirazione per i briganti, le ricorrenti, sterili insurrezioni, l'immutabilità dei rapporti sociali rivelano che il cosiddetto problema meridionale è in realtà $i l$ problema della comunità costituzionale italiana. Sottratto alle sue comodità borghesi, e gettato nella peripheral view del mondo dei contadini e degli emarginati, Levi arriva a conquistare una visione politica autenticamente federale.

Tuttavia il suo racconto si inserisce nella storia della cultura giuridica e politica italiana, in cui le narrazioni federali sono sempre state minoritarie e contro-egemoniche. L'occasione del periodo costituente non venne colta dalle forze politiche ed il regionalismo debole della costituzione repubblica ha mostrato tutte le sue insufficienze. Per una prospettiva di "diritto e 
letteratura" dunque, il Cristo si è fermato a Eboli non è solo un capolavoro della narrativa (La Porta, 2001, p. 140) - e dopo la sua eccellente trasposizione cinematografica di Francesco Rosi nel 1979, anche della filmografia (Lerner, 2009, p. 272; Fochi, 2009, p. 167) $)^{2}$, ma è anche un testo classico della tradizione federalista italiana (Lupo, 1998, p. 33; Marmo, 2005, p. 175).

Per concludere vorrei evidenziare i vari tipi di censure che si incontrano nel Cristo: vi è innanzitutto la censura politica, del regime fascista nei confronti dell'attivista politico Carlo Levi, che risulta in arresti, detenzioni e nel confino politico, che paradossalmente finì per "liberare" il dottore/pittore dal suo narcisismo e permettergli di dispiegare la sua umanità empatica più profonda, nei rapporti con il mondo contadino.

Vi è poi la censura del sindaco di Aliano e del prefetto di Matera, che controllano la corrispondenza del confinato, ne inibiscono la cronaca immediata e rendono possibile l'incubazione dei ricordi vitali, destinati a maturare nella scrittura solo otto anni dopo.

Nel romanzo vi è poi rappresentata la censura del mondo piccoloborghese nei confronti dei contadini, in un rapporto di odio e sfruttamento, senza comunicazione autentica né comprensione umana. Vi è poi la censura della Weltgeschichte, che prima costringe un ebreo laico alla fuga in Francia, gli impedisce nel ' 41 di imbarcarsi per gli Stati Uniti, lo spinge a rientrare in Italia con il regime ancora al potere e con le leggi razziali ancora in vigore, e gli permette poi, in condizioni di clandestinità, di scrivere e di "riandare con la memoria a quell'altro mondo, serrato nel dolore e negli usi, negato alla Storia e allo Stato, eternamente paziente” (Levi, 2014, p. 3), producendo così uno dei capolavori della letteratura mondiale.

Ma vi è infine un altro tipo di censura, meno violenta ma non per questo meno efficace, costituita dall'incomunicabilità tra la visione federale organica di Levi e la persistente adorazione della teocrazia statale da parte degli intellettuali e dei politici vicini a Levi. La censura soft che la cultura centralistica ha esercitato sulle concezioni federaliste nella storia politica dell'Italia unita non si è purtroppo arrestata al tempo del 1935, ma è

2 Lo sceneggiato televiso del 1980 può ora essere visto qui: http://www.raiplay.it/programmi/cristosiefermatoaeboli/ 
proseguita nell'occasione perduta della stagione costituente e, aimè, giunge sino ai nostri giorni.

Rileggendo Cristo si è fermato a Eboli, si vede come il linguaggio giuridico delle istituzioni locali, emanazione dello stato centrale, con il suo individualismo formalista ha costituito un dispositivo di esclusione nei confronti del mondo contadino. Nel periodo monarchico della storia italiana le minoranze cittadine hanno parlato un linguaggio incomprensibile alle maggioranze della campagna. Ma anche il linguaggio delle élites politiche repubblicane è rimasto impermeabile al discorso contro-egemonico dei federalisti. In Italia il logos centralistico ha censurato tanto il mondo di vita contadino, marginale e periferico, quanto le possibilità trasformatrici della visione federalista, mantenuta nella dimensione utopica, potenzialmente rivoluzionaria, ma di fatto incapace di tradursi in mutamento della cultura costituzionale dominante.

\section{RIFERIMENTI}

BARTALESI-GRAF, Daniela. “Torino" e "Matera” nella prosa e nell'arte di un anno di confino. Italica, v. 83, n. 3-4, p. 505-528, Fall-Winter, 2006.

COCCIA, Nicola. L'arse argille consolerai. Pisa: Edizioni ETS, 2015.

DE DONATO, Gigliola; D'AMARO, Sergio. Un torinese del Sud: Carlo Levi; una biografia. Milano: Bladini \& Castoldi, 2005.

LERNER, Giovanna Faleschini. Francesco Rosi's Cristo si è fermato a Eboli: Toward a Cinema of Painting. Italica, v. 86, n. 2, p. 272-292, Summer, 2009.

FOCHI, Anna. Oltre Eboli: il viaggio di Rosi nell'universo remoto del "Cristo" di Levi. Italianistica - Rivista di letteratura italiana, v. 38, n. 3, p. 167-176, sett./dic. 2009.

HÄBERLE, Peter. Demokratische Verfassungstheorie im Lichte des Möglichkeitsdenken, in Verfassung als Öffentlicher Prozess. Berlin: Duncker \& Humblot, 1996.

KING, Russell; KILLINGBECK, Jenny. Carlo Levi, the Mezzogiorno and Emigration: Fifty Years of Demographic Change at Aliano. Geography, v. 74, n. 2, p. 128-143, Apr. 1989.

LA PORTA, Filippo. Carlo Levi e la nuova narrativa meridionale. Meridiana, n. 40, p. 137-143, 2001.

LEVI, Carlo. Cristo si è fermato a Eboli. Torino: Einaudi, 2014.

LUPO, Salvatore. Storia del Mezzogiorno, questione meridionale, meridionalismo. Meridiana, n. 32, p. 17-52, giugno 1998.

MARMO, Marcella. Discutendo di autonomia da Carlo Levi a oggi. Meridiana, n. 53, p. 149-186, 2005. 
RAGUSA, Olga. Review of "Cristo si è fermato a Eboli". Italica, v. 46, n. 3, p. 336-337, Autumn, 1969.

ROMANO, L. Carlo Levi. Un'esperienza culturale e politica nella Torino degli anni Trenta. Torino: Archivio di Stato di Torino, 1985.

VESPAZIANI, Alberto. Federalismo. In: Diritto on line. Disponibile in: $<$ http://www.treccani.it/enciclopedia/federalismo-dir-cost (Diritto-online)/>. Accesso: 20 Dic. 2016.

Lingua originale: Italiano

Ricevuto: 06/02/16

Accettato: 12/06/16 